\title{
IoT based poultry house monitoring
}

\begin{abstract}
Recently, the use of IoT (Internet of Things) based system has been expanded with inestimable Internet resources. The system demonstrates the creation of innovative systems that facilitate control and supervision regardless of distance and time. In a poultry house, both temperature and humidity levels should be monitored regularly in ensuring the system runs smoothly. It needs to be monitored 24/7 to avoid incidents that caused the temperature rises too high. This paper highlights the IoT solution in monitoring the temperature and humidity condition including the presence of electricity connectivity, regardless of time and place.
\end{abstract}

Keyword: Embedded system; Raspberry Pi; IoT; Mobile apps 